\title{
Politique
}

Politique

\section{Alain Baccigalupo, avec la collaboration de Luc Rhéaume, Les administrations municipales québécoises des origines à nos jours, Tome 1, Les municipalités, Montréal, Agence d'Arc, 1984.}

\section{Maurice Couture}

Numéro 8, automne 1985

Innovations et politiques technologiques

URI : https://id.erudit.org/iderudit/040507ar

DOI : https://doi.org/10.7202/040507ar

Aller au sommaire du numéro

Éditeur(s)

Société québécoise de science politique

ISSN

0711-608X (imprimé)

1918-6584 (numérique)

Découvrir la revue

Citer ce compte rendu

Couture, M. (1985). Compte rendu de [Alain Baccigalupo, avec la collaboration de Luc Rhéaume, Les administrations municipales québécoises des origines à nos jours, Tome 1, Les municipalités, Montréal, Agence d'Arc, 1984.] Politique, (8), 165-168. https://doi.org/10.7202/040507ar d'utilisation que vous pouvez consulter en ligne. 
Alain Baccigalupo, avec la coilaboration de Luc Rhéaume, Les administrations municipales québécoises des origines à nos jours, Tome 1, Les municipalités, Montréal, Agence d'Arc, 1984.

C'est avec un grand intérêt et beaucoup d'attente que j'ai examiné l'ouvrage d'Alain Baccigalupo qui avoue dès le premier paragraphe introductif son «ambition (...) de publier un ouvrage dont la critique dira, selon la formule consacrée, qu'il vient à point combler une très importante lacune».

Je dirai tout de suite, pour me situer, qu'en effet, il vient à point, mais la très importante lacune demeure.

Il vient à point: ceux qui œuvrent dans le domaine municipal - enseignants, chercheurs et praticiens de tous ordres - fonctionnaires centraux, locaux et élus locaux - ont eu à faire face 
à une législation abondante et importante depuis une décennie. Ils s'attendaient à ce que vienne un ouvrage qui tente de jeter une certaine lumière sur le niveau municipal. L'auteur a osé faire un pas. Il faut lui en attribuer le mérite. Cependant, force est d'admettre que le flux lumineux n'a pas su traverser les ténèbres créées par ce que je viens d'appeler l'abondante et importante législation dans le secteur municipal. Je passerai donc en revue chacun des chapitres pour montrer en quoi l'ouvrage ne sert pas bien les «clients» déjà identifiés.

Disons d'abord que l'organisation de l'ouvrage montre que l'auteur a su percevoir la réalité de l'administration municipale et répondre à certains besoins des acteurs impliqués dans le milieu. Cela constitue un bon départ pour une autre édition améliorée. Mais revenons à chacune des parties.

Un premier chapitre sur l'histoire des administrations municipales à sa place dans ce type d'ouvrage mais en occupe trop dans celui-ci: 100 pages sur 600. Il s'agit là d'une partie déjà bien traitée et trop connue des spécialistes et d'une utilité relative pour les novices. Le traitement qu'il en fait, c'est-à-dire les textes choisis ne permettent pas de dégager les temps des trois différents régimes présentés par l'auteur. Par ailleurs, la prétention de faire remonter les institutions centrales de la Nouvelle-France à la Compagnie des cent associés me paraît exagérée. Voulant faire original, je crois que l'auteur force un peu l'histoire.

Le deuxième chapitre nous introduit au domaine crucial des structures administratives selon trois approches qui annoncent un traitement fort pertinent de cette question: l'approche sociologique, l'approche institutionnelle et l'approche conceptuelle. Cependant leur traitement s'avère très inégal et décevant. Les six pages consacrées à la première se ramènent à trois si on exclut tableaux et graphiques. Si l'on ajoute à cela l'interprétation douteuse que l'auteur fait du régressement urbain du Québec fondé, selon lui, sur «le départ de nombreux anglophones de la région métropolitaine de Montréal 
en direction des provinces de l'Ouest» (p. 125), cette section constitue un raté.

La deuxième qui fait état de la diversité des sources du droit municipal et de la complexité des catégories de municipalités se voit bien traitée alors que la dernière, pleine de promesses, se résume à la présentation de six définitions tirées de recueils de jurisprudence.

La partie la plus réussie de l'ouvrage porte sur les élus locaux. Le processus électoral et l'exercice des fonctions électives municipales constituent deux domaines fort transformés par la législation récente. Or, rassembler les diverses dispositions législatives dans ces cas permet une vue nette de la réalité actuelle. Ce que l'auteur a bien réalisé en proposant des extraits importants des lois souvent modifiées. Évidemment, rien de nouveau pour les lecteurs de la Revue Municipalité, mais utile pour les autres. Ce chapitre se dirigeait vers l'excellence jusqu'à ce que dans la conclusion l'auteur nous entraîne dans un débat un peu trivial, extrait du quotidien $L e$ Soleil, sur la juridiction de la fonction de maire.

Je n'hésite pas à exprimer ma déception à l'égard du $4^{\mathrm{e}}$ chapitre sur les fonctions publiques municipales. Avec les structures et les finances, c'est un sujet de première importance. Or les extraits traitant du personnel municipal salarié proviennent d'une recherche bien faite mais partielle et qui remonte à plus d'une décennie, et de la traduction d'un classique américain, sérieux, mais également daté peu utilisable dans notre contexte.

Quant à la seconde partie de ce chapitre traitant des officiers municipaux, toute la section concernant le gérant municipal s'avère quasi inutile dans la mesure où une loi adoptée déjà lors de la parution de l'ouvrage en a changé à la fois les prérogatives et l'appellation. On parle maintenant de directeur général.

Un cinquième chapitre, important aussi, traitant des services et équipements municipaux se voit offrir un bon début de traitement. L'auteur fait l'effort d'introduire les catégorisations classiques de 
l'administration publique «traditionnelle ou gendarme» et «moderne ou providence». Le problème est qu'il s'arrête là. N'importe qui aurait attendu un début de débat qui pourtant n'a rien de nouveau sur les tendances des administrations à se "privatiser». Les théoriciens du "public choice» ont puisé tant d'exemples dans le domaine municipal et les gestionnaires municipaux n'ont pas hésité à faire des expériences, qu'il eût été facile de trouver des textes pour compléter ce chapitre.

Enfin le dernier chapitre sur les finances municipales, en reprenant les grands débats des commissions d'enquête sur le sujet et le projet devenu réalité de la réforme fiscale municipale constitue lui aussi un apport fort utile. Bien qu'il s'agisse d'un secteur fort controversé, les extraits importants des documents gouvernementaux récents donnent une idée juste de l'état de la question au Québec. Bien que, encore, le débat névralgique sur la tarification à l'usager n'ait pas trouvé de place.

Il m'est difficile de recommander l'utilisation d'un tel ouvrage qui, malgré les efforts exigés pour donner des outils à ceux qui veulent comprendre le monde municipal donne tant de place à des textes secondaires et néglige des travaux sérieux. Des travaux de chercheurs reconnus ne sont jamais cités. C'est le cas des meilleurs. Ainsi il m'apparaît qu'il s'agit davantage d'un spicilège que d'une anthologie.

Maurice Couture U.Q.A.M. 